\title{
Age-Structured Model for COVID-19: Effectiveness of Social Distancing and Contact Reduction
}

\section{Mark Kimathi ( $D$ mark.kimathi@mksu.ac.ke)}

Machakos University College https://orcid.org/0000-0002-5822-8687

\section{Samuel Mwalili}

Jomo Kenyatta University of Agriculture and Technology

Viona Ojiambo

Jomo Kenyatta University of Agriculture and Technology

\section{Duncan Gathungu}

Jomo Kenyatta University of Agriculture and Technology

\section{Research article}

Keywords: mathematical model, coronavirus, age structured, contact matrix, non-pharmaceutical intervention

Posted Date: June 29th, 2020

DOI: https://doi.org/10.21203/rs.3.rs-33480/v1

License: (1) (1) This work is licensed under a Creative Commons Attribution 4.0 International License. Read Full License

Version of Record: A version of this preprint was published at Infectious Disease Modelling on January 1st, 2021. See the published version at https://doi.org/10.1016/j.idm.2020.10.012. 


\title{
Age-Structured Model for COVID-19: Effectiveness of Social Distancing and Contact Reduction
}

\author{
Mark Kimathi??????, Samuel M Musili??, Viona Ojiambo??, Duncan K Gathungu??
}

\footnotetext{
?? Correspondence:

?? Department of Mathematics and Statistics, Machakos University, Machakos, Kenya Full list of author information is available at the end of the article

?? Equal contributor
}

\begin{abstract}
Background: Coronavirus disease 2019 is caused by severe acute respiratory syndrome coronavirus 2 . The disease has spread to almost every country in the world. Kenya reported its first case on 13th of March 2020. From 16th March 2020, the country instituted various social distancing strategies to reduce the transmission and flatten the epidemic curve. These strategies include school closure, dusk-to-dawn curfew, and travel restriction across counties, especially Nairobi, Mombasa and Kwale. An age-structured compartmental model was developed to assess the impact of non-pharmaceutical interventions on severity of infections, hospital demands and deaths.
\end{abstract}

Methods: The population is divided into four age-groups and for each age-group there are seven compartments, namely: susceptible, exposed, asymptomatic, mild, severe, critical, death and recovered. The contact matrices between the different ages are integrated into an age-structured deterministic model via the force of infection. This model is represented by ordinary differential equations and solved using Runge-Kutta methods, with suitable model parameters. Simulation results for the unmitigated and mitigated scenarios were depicted, for the different age-groups.

Results: The $45 \%$ reduction in contacts for 60 -days period resulted to between $11.5-13 \%$ reduction of infections severity and deaths, while for the 190 -days period yielded between $18.8-22.7 \%$ reduction. The peak of infections in the 60 -days mitigation was higher and happened about 2 months after the relaxation of mitigation as compared to that of the 190-days mitigation, which happened just a month after mitigation were relaxed. Low numbers of cases in children under 15 years was attributed to low susceptibility of persons in this age-group. High numbers of cases are reported in the 15-29 years and 30-59 years age bands since these individuals have wider interaction spheres, and they form a significant percentage of Kenya population.

Conclusion: Two mitigation periods, considered in the study, resulted to reductions in severe and critical cases, attack rates, hospital and ICU bed demands, as well as deaths, with the 190-days period giving higher reductions. The study revealed the age-dependency of the key health outputs.

Keywords: mathematical model; coronavirus; age structured; contact matrix; non-pharmaceutical intervention

\section{Background}

The coronavirus disease 2019 (COVID-19) is caused by severe acute respiratory syndrome coronavirus 2 (SARS-CoV-2). The first reported case was in mainland 
China, City of Wuhan, Hubei on the 29th of December 2019[?]. Subsequently the disease spread at an exponential rate to countries in contact with China resulting to World Health Organization (WHO) declaring it as a Public Health Emergency of International Concern (PHEIC) on 30th January 2020[?]. As of 31st May 2020 there were over six million infections globally, with the European region taking lead in these infections [?]. In Africa, the first case was reported in Egypt then followed by Algeria [?]. The first Kenyan case was reported on the 13th of March and by 31st May 2020 there were about 1,900 confirmed cases, with Nairobi and Mombasa taking the lead in these infections[?].

There are mainly three kinds of infections; asymptomatic, pre-asymptomatic and symptomatic. The incubation period for COVID-19, which is the time between after exposure to the virus and symptoms onset, is on average 5-6 days, however it can be as high as 14 days. For COVID-19 symptomatic case, the disease manifests itself through symptoms such as fever, coughs, sneezes and headaches, whereas for asymptomatic case the infected individual does not develop symptoms[?]. The basic reproduction number, defined as the average number of secondary infections produced by an infectious individual in a population where everyone is susceptible [?], is affected by the rate of contacts in the host population, the probability of transmission during contact and the duration of infectiousness. It can also vary for different age bands since the attack rates are age-dependent. The basic reproduction number for COVID-19 in Kenya ranges from 1.78(95\%CI1.44 - 2.14) to $3.46(95 \%$ CI2.81 - 4.17)[?]. Reduction of the reproduction number can definitely be achieved by instituting appropriate Non-Pharmaceutical Interventions (NPIs) or use of a vaccine.

In the absence of a vaccine, social/physical distancing strategies have globally become the most appropriate Non-Pharmaceutical Interventions (NPIs) [?]. These mitigation can be implemented by reducing social contacts in workplaces, schools, markets and other public areas. Social contacts are influenced by age structure of the population and the frequency of contacts across population [?]. Mathematical models that describe the impact of the NPIs in reducing morbidity, infection peak sizes, and excess mortality are vital in public-health planning [?].

Similar to other sub-Saharan African countries, the Kenyan government has imposed travel restrictions across counties, dusk-to-dawn curfew and school closure to ensure social distancing in the population and consequently slow transmission of COVID-19. Although it is not clear for how long these measures should be in place to eradicate the epidemic in Kenya, we state that premature and sudden lifting of interventions could potentially lead to a new peak of infections. However, intermittent application of the interventions can flatten the infections curve [?]. Previous study of COVID-19 in Kenya also predicted the risk of epidemic rebound after the social distancing measures are lifted [?].

In this study, an age-structured SEIR mathematical model that examines the impact of NPIs in curbing COVID-19 severity and deaths in Kenya is developed, with the aim of achieving the following; (i) assessing the impact of reducing social contacts in different age-groups,(ii) examine the trend in infections during and after the NPIs,(iii) provide plausible period for lifting the NPIs. We postulate that this 
study can form a basis for policy formulation to enable Kenya delay the disease transmission and eventually flatten the epidemic curve.

\section{Methods}

The Kenya population is split into the four broad age groups [?]: those below 15 years, $15-29$ years, $30-59$ years, and above 60 years. These are denoted by subscript $i=1,2,3,4$, respectively. Each population of age group $i$ is classified as either susceptible $S_{i}$, exposed $E_{i}$, asymptomatic $A_{i}$, mild $M_{i}$, severe $H_{i}$, critical $C_{i}$ or recovered $R_{i}$. The sum of the compartments $N_{i}$ gives the size of the population in age group $i$.

$$
N_{i}=S_{i}+E_{i}+A_{i}+M_{i}+H_{i}+C_{i}+R_{i}
$$

The exposed $E_{i}$ compartment represents the individuals infected with coronavirus but are not yet infectious, since the virus is in incubation stage. The asymptomatic $A_{i}$ compartment has those individuals who are infectious, but do not exhibit the disease symptoms. The mild $M_{i}$ compartment has infectious individuals who exhibit symptoms of COVID-19, but their condition does not require hospitalization. The severe $H_{i}$ compartment has infected individuals who needs to be hospitalized so as to manage their condition better. Finally, the critical $C_{i}$ compartment contains infected individuals whose situation is much worse as compared to a severe case, in that they are required to be in intensive care unit. The susceptible individuals are exposed/infected through contact with infectious individuals from any of the age-groups. After the disease incubation period, exposed individuals progress into either the asymptomatic or mild compartment. Infectious individuals who are asymptomatic, are assumed to recover over time whereas the mild cases either recover or progress to the severe compartment. Depending on the disease severity, the individuals in $H_{i}$ either recover or become critically ill. These critical cases, now in $C_{i}$ either die or their condition improves to a severe case, no longer requiring ventilation. The dynamics of the epidemic in our age-structured model is governed by the flow diagram in Figure ??. The flow diagram yields the following model equations:

$$
\begin{array}{r}
\frac{d S_{i}}{d t}=\left(-\beta_{1, i}(t)-\beta_{2, i}(t)\right) S_{i}, \\
\frac{d E_{i}}{d t}=\left(\beta_{1, i}(t)+\beta_{2, i}(t)\right) S_{i}-\omega E_{i}, \\
\frac{d A_{i}}{d t}=\theta_{i} \omega E_{i}-\gamma_{A, i} A_{i}, \\
\frac{d M_{i}}{d t}=\left(1-\theta_{i}\right) \omega E_{i}-\left(\kappa_{i}+\gamma_{M, i}\right) M_{i}, \\
\frac{d H_{i}}{d t}=\kappa_{i} M_{i}+\phi_{i} C_{i}-\left(\xi_{i}+\gamma_{H, i}\right) H_{i}, \\
\frac{d C_{i}}{d t}=\xi_{i} H_{i}-\left(\phi_{i}+\lambda_{i}\right) C_{i}, \\
\frac{d R_{i}}{d t}=\gamma_{A, i} A_{i}+\left(\gamma_{M, i}+\gamma_{H, i}\right) M_{i} .
\end{array}
$$

Description of the age-dependent model parameters are presented in the Table ??. Human-human transmission of coronavirus depends on whom one is in contact 
with and where. The place of contact could be at home, school, work, or within the community e.g. markets, restaurants etc. Therefore, we assume the susceptible individuals will acquire the virus when they come into contact with an infectious individual, and express the rate of infections $\beta_{1, i}(t)$ and $\beta_{2, i}(t)$ as follows:

$$
\begin{gathered}
\beta_{1, i}(t)=\beta_{1} \sum_{j=1}^{4} C_{i j} \frac{A_{j}(t)}{N_{j}}, \\
\beta_{2, i}(t)=\beta_{2} \sum_{j=1}^{4}(1-\alpha) C_{i j} \frac{M_{j}(t)}{N_{j}},
\end{gathered}
$$

for $i=1,2,3,4$. $C_{i j}$ denotes contact matrices and describe the interactions between the considered age-group $i$ with other age-groups $j$. The constants $\beta_{1}$ and $\beta_{2}$ represent the likelihood of infection upon contact, and based on the basic reproduction number for COVID-19. The parameter $\alpha \in[0,1]$ denotes the proportion of mild (symptomatic) infectives who self-isolate to minimize their contacts, which is a control measure encouraged by the health experts during the coronavirus pandemic.

\section{Specification of the contact matrix}

Contact matrix $C_{i j}$ comprises of contacts at home $(H)$, workplace $(W)$, school $(S)$ and other $(O)$ contact which is not happening at home, work or in school. Therefore we express $C_{i, j}$ as follows:

$$
C_{i j}=C_{i j}^{H}+C_{i j}^{W}+C_{i j}^{S}+C_{i j}^{O}
$$

where for instance the home contact matrix $C_{i j}^{H}$, for $i, j=1,2,3,4$ is expressed as follows:

$$
C_{i j}^{H}=\left[\begin{array}{cccc}
C_{11}^{H} & C_{12}^{H} & C_{13}^{H} & C_{14}^{H} \\
C_{21}^{H} & C_{22}^{H} & C_{23}^{H} & C_{24}^{H} \\
C_{31}^{H} & C_{32}^{H} & C_{33}^{H} & C_{34}^{H} \\
C_{41}^{H} & C_{42}^{H} & C_{43}^{H} & C_{44}^{H}
\end{array}\right],
$$

such that the matrix elements range between 0 and 1 ; with value 0 implying no contact and value 1 implying maximum contact. During the coronavirus epidemic the contact patterns are definitely not the same as compared to the no epidemic times [?].

\section{Implementation of Social Distancing Strategies}

The mixing of different age-group populations has been incorporated in our model equations through contact matrices, (??) which are used in (??). We now describe the reduction of contacts in schools, workplaces, and the general community caused by the social distancing measures by a time-dependent control:

$$
u(t)=1-e *\left(\tanh \left(\frac{t-t_{o n}}{t_{w}}\right)+\tanh \left(\frac{t-t_{o f f}}{t_{w}}\right)\right),
$$

where $t_{o n}$ and $t_{\text {off }}$ are respectively the day of imposing and lifting the control measure. $t_{w}$ is a shape parameter whereas the constant $E$ is chosen such that the 
desired reduction in contact is achieved. We apply this function to the non-household contact matrices to obtain:

$$
C_{i j}(t)=C_{i j}^{H}+u(t)\left(C_{i j}^{W}+C_{i j}^{S}+C_{i j}^{0}\right)
$$

Using (??) in (??) enables us to implement the interventions of school closure, dusk-to-dawn curfew, and movement restriction independently and at the precise time they were instituted. Closure of schools yields a $100 \%$ reduction in the school contacts, as such $C_{i j}^{S}$ will be a matrix of zeros. Assuming that imposing a dusk-todawn curfew and restricting movement in and out of hotspots reduces the social mixing in workplaces and in other places (besides home, school, and work) by $35 \%$ and $45 \%$ respectively we obtain the contact patterns depicted in Fig. ??. The actual contact matrices for Kenya were unavailable to the authors, so we produced synthetic contact matrices guided by [?] and [?]. Panels (A), (B), and (C) depicts the contacts at workplaces between working age groups. Panel (B) show a $35 \%$ reduction in contacts at work due to the time constraints brought about by the dusk-to-dawn curfew, in which the working hours are reduced to allow individuals get home before dusk.

When the movement restriction is instituted for non-essential services, whereby people are not allowed to travel in and out of certain regions, we see much less contacts at work in panel (C). Panels (D), (E), and (F) show contacts which are dominant along the diagonal and in age groups less than 50 years. These contacts are happening in places that are not work, school or home. Therefore, they constitute contacts in marketplaces, entertainment places, or other social gatherings such as weddings. Hence the mixing is highly assortative and is likely to bring into contact individuals of same age groups but from distant regions. Therefore, it is imperative to control interactions in this category of contacts, otherwise the epidemic would spread very fast in the communities. As shown in panels (E) and

$(\mathrm{F})$, the social distancing measures imposed on $C_{i j}^{0}$ through (??) are effective in minimizing these contacts by $35 \%$ and $45 \%$ respectively. In Africa, majority of the population is generally less than 35 years, and often in contact with children and their (grand)parents as indicated by the main diagonal and off-diagonals in panels $(\mathrm{G}),(\mathrm{H})$, and $(\mathrm{I})$. Noting that ministry of health is advising people to stay at home, we postulate that imposing the curfew and movement restriction increases home contacts by $12.5 \%$ and $25 \%$ respectively, as shown in panels (H) and (I).

\section{Simulation Set-up}

To show the impact of the highlighted measures in Kenya, we present results for daily and cumulative infections, severe and critical cases, deaths, as well as peak demand for hospital and ICU beds. The simulation was done for a one year starting from 13th March, 2020, but we present results for upto December, 2020 since the evolution of the epidemic after this period is subject to uncertainties. To initialize the simulation, we assumed $A_{1}(0)=1, M_{1}(0)=1, M_{2}(0)=1, M_{3}(0)=2$, and $M_{4}(0)=1$ while all other compartment populations were taken as zero, but $S_{i}(0)=$ $N_{i}-E_{i}(0)-A_{i}(0)-M_{i}(0)-H_{i}(0)-C_{i}(0)-R_{i}(0)-D_{i}(0)$. The populations $N_{1}$, $N_{2}, N_{3}$, and $N_{4}$ are respectively obtained as $39 \%, 28 \%, 28 \%$, and $5 \%$ of $4.76 \times 10^{6}$, 
Kenya's total population. The parameter values used are presented in Table ?? For the unmitigated scenario we assumed $R o=2.5$, which is within the range in the study [?], of COVID-19 in Kenya. The transmissions of infection were obtained from (??), where the contact matrices are implemented through (??). The time lag (in days) before the instituted social measure took effect was assumed to be 1.5 days, and added to $t_{\text {on }}$ value. The schools were closed in Kenya only three days after the first confirmed case of COVID-19 i.e. on 16th March, 2020. Then on 27 March, 2020 the government imposed a dusk-to-dawn curfew, and on 7th April, 2020 travel was restricted in/out of Nairobi, Mombasa, Kilifi and Kwale counties [?]. These dates informed the start date for the three interventions, which we let to be in place for 60 days and 190 days respectively, before they are all lifted at once. We assumed the infectiousness of asymptomatic individuals to be $33 \%$ while the symptomatic individuals to be $67 \%$. Finally, we assume that $30 \%$ of confirmed symptomatic individuals will self-isolate [?] to reduce their contact with susceptible population i.e. $\alpha=30 \%$. This control measure is uniform across all the age-groups and assumed part of Kenya's mitigation efforts along side the school closure, curfew and travel restrictions.

\section{Results and Discussion}

\section{Simulation Results of Effects of Social Distancing Measures}

The simulation results are depicted in Figure ?? and Table ??. In Figure ??, the duration of school closure is indicated by cyan shaded region and is overlapped by the duration of implementing the dusk-to-dawn curfew indicated by yellow shaded region. The gray shaded region indicate duration of implementing travel restriction across counties. The interventions begin at different days but they all end at the same day, as shown by the light-gray region for the 60-days mitigation and darker-gray for the 190-day mitigation. The social distancing measure lasting for 60 days resulted to a delay of the epidemic peak for about 2 months compared to the unmitigated situation which peaked within 5255 days. The $45 \%$ reduction in contacts for 60 days resulted to between 11.5$13 \%$ reduction of cumulative infections. When the social distancing measures were in place for 190 days the epidemic peak was delayed for about 5 months compared to the unmitigated scenario. Also the $45 \%$ reduction in contacts for the 190 days resulted to between $18.8-22.7 \%$ reduction of cumulative infections.

The peak of infections in the 60-days mitigation is higher and happens about 2 months after the mitigation is relaxed as compared to that of the 190-days mitigation, which happens a month after mitigation is relaxed. This is due to insufficient herd-immunity since the infections are quite suppressed during the 60 days as compared to significant presence of infections for the 190-days mitigation before the measures are relaxed, as shown in Figure ??. Also shown is a notable rise in infections after the interventions are lifted. However, due to herd-immunity and the depletion of susceptible in the population the rise in infections is not sustained.

\section{Simulated Severe, Critical Cases, Hospital Demands and Deaths}

From Table ?? we show the age dependence in the simulated cases and peaks. In all the cases presented in the table, the numbers for those under 15 years are low. 
This is the age group with a high number of asymptomatic infections, which are more likely to remain undetected although they are still transmitting the virus, and bearing in mind that children make more social contacts as compared to adults. The low numbers could also be due to age-varying susceptibility to COVID-19 infections, whereby the children could be less susceptible upon being in contact with an infectious individual. High number of cases are reported for the 15-29 years and 30-59 years age bands since majority of individuals in these age bands have wider interaction spheres (outside of schools and home), and they form a significant percentage of Kenya population. The considered mitigation periods yielded reductions in the key health outputs, although applying the mitigation for entire simulation time of 365 days would have resulted into more significant reductions. However, in reality the population might not withstand the long-term imposing of dusk-to-dawn curfew and travel restrictions. The high numbers of severe and critical cases translates to high demands for hospital and ICU beds, and also deaths. In the 190-day mitigation in Table ?? there is an increase in hospital and ICU beds peak demands which is likely due to the notable rise in infections after the measures have been relaxed, as shown in Figure ??.

\section{Simulated Attack Rates}

The overall and symptomatic attack rates are presented in Table ?? and they exhibit age-dependency. The younger population have lower attack rates (and lower epidemic peak sizes) as compared to the older population whereby those older than 59 years have the highest overall attack rate, as well as the highest symptomatic attack rate. This result shows the age-dependency of exposed individuals progressing to symptomatic cases. The 190-days mitigation period reduces the attack rates and subsequently flattens the epidemic curve. However imposing these stringent measures for a prolonged period has adverse effects on the socio-economics of the country. The dependency of the attack rates on age underscores the variability of $R_{o}$ across the age bands [?].

\section{Conclusion}

The dependency of COVID-19 transmissions, severity and deaths on age is crucial to the design of social distancing measures and projection of the expected disease burden in the country. Indeed the considered interventions do not completely avert the epidemic, but they significantly slow down the transmissions and reduce the infection peak sizes, and deaths. We note that if there is no self-isolation of symptomatic cases, the number of cases and deaths will increase, which will result to the peaks happening earlier in all cases. Prolonged implementation of social distancing measures will definitely resolve the epidemic, however it will damage the country economically. It is not fully known how the epidemic would spread to various counties in Kenya, and how people in these counties will react to the NPIs. There is need for coordination and frequent exchange of information between modeling and surveillance groups in order to refine predictions of the epidemic trajectory.

\section{Abbreviations}

COVID-19: Coronavirus disease 2019; SARS-CoV-2: Severe acute respiratory syndrome coronavirus 2; WHO: World health organization; PHEIC: Public health emer- 
gency of international concern; NPIs: non-pharmaceutical interventions; SEIR: Susceptible exposed infectious recovered; ICU: Intensive care unit; CDC: Centers for disease prevention and control.

\section{Declarations}

Ethics approval and consent to participate Not applicable.

Consent for publication

Not applicable.

Availability of data and material

The data used in this study was publicly available.

Competing interests

The authors declare that they have no competing interests.

Funding

This study has not received any funding.

Author's contributions

All the authors equally contributed to this article.

Acknowledgments

The authors appreciate the valuable advice offered by Peter Young and Thomas Achia of Centers for Disease Prevention and Control (CDC), Kenya.

Figures

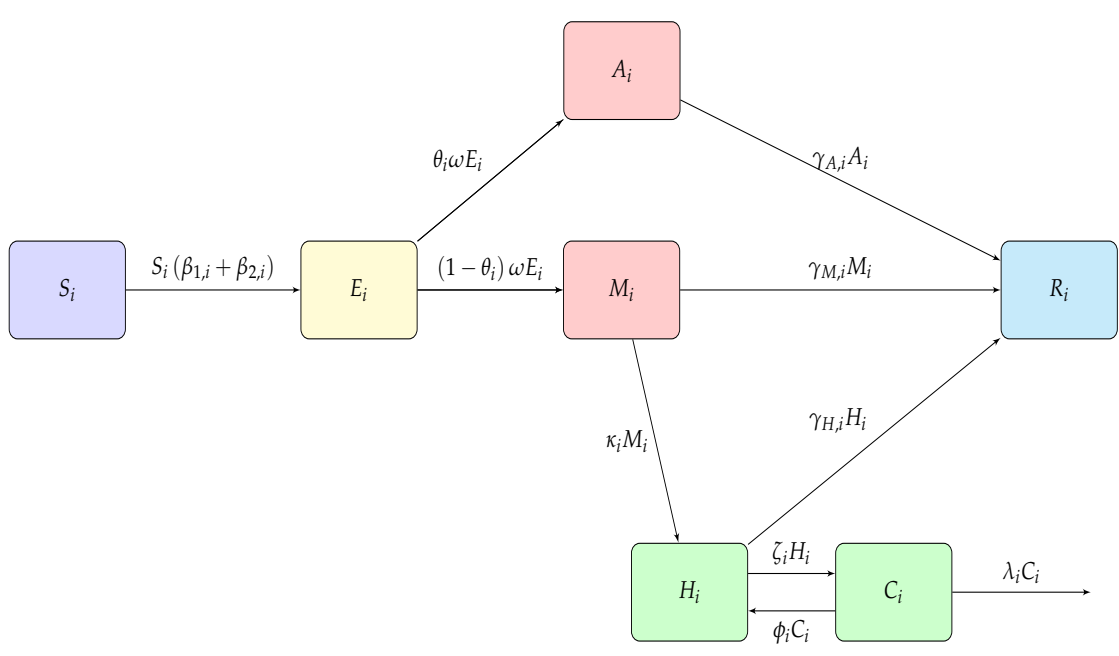

Figure 1 Flow diagram of the age-structured model for COVID-19 incorporating disease severity in the infected individuals 


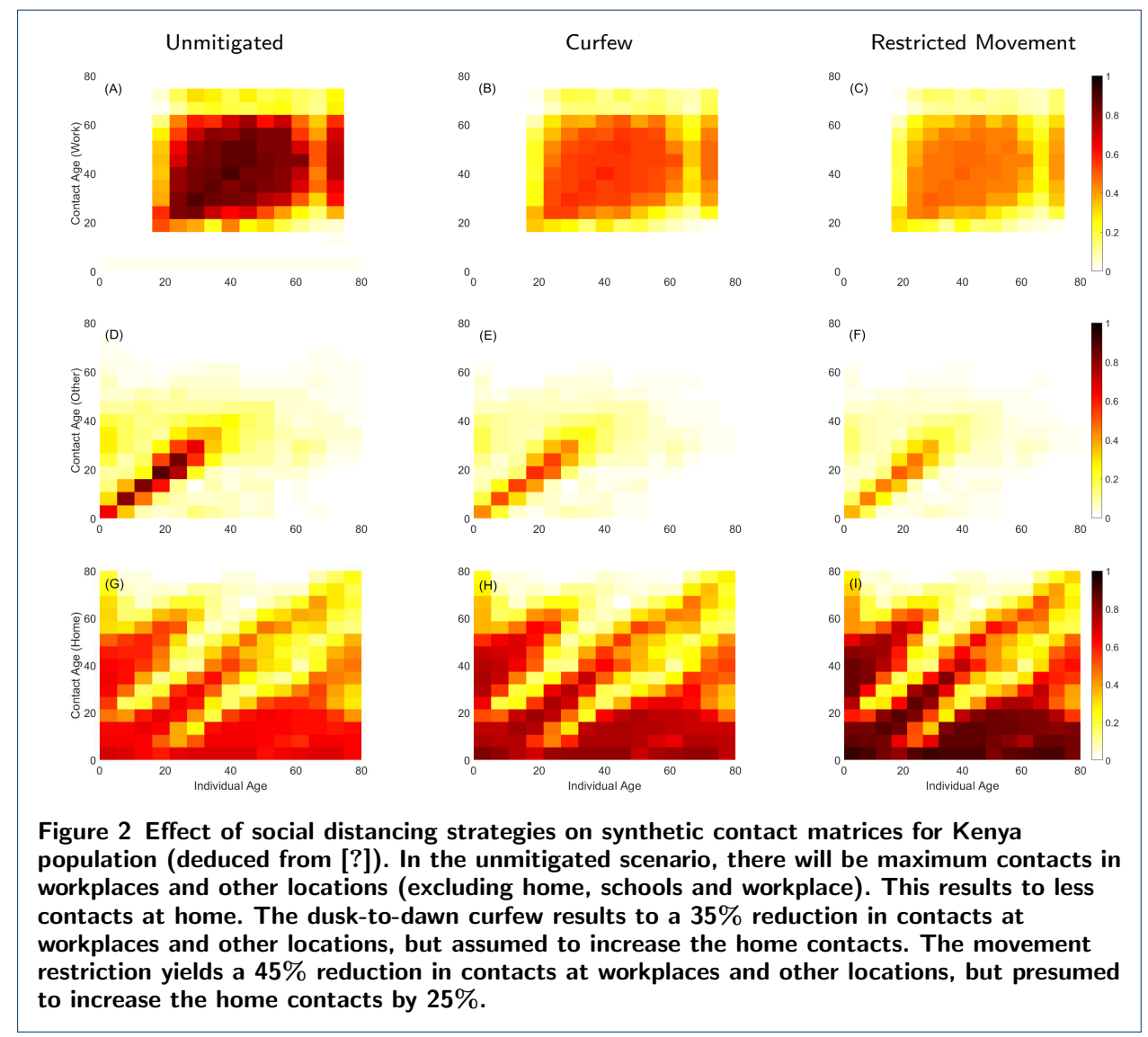

Table 1 Description of model parameters and their values. The age-specific parameter values are determined based on the references [?],[?],[?],[?]

\begin{tabular}{l|l|l|l|l|l|}
\hline Model Parameter Name & Symbol & $\begin{array}{c}\text { below 15 } \\
\text { years }\end{array}$ & $\begin{array}{c}\mathbf{1 5 - 2 9} \\
\text { years }\end{array}$ & $\begin{array}{c}\mathbf{3 0}-\mathbf{5 9} \\
\text { years }\end{array}$ & $\begin{array}{c}\text { above 59 } \\
\text { years }\end{array}$ \\
\hline Proportion of Asymptomatic (A) & $\theta_{i}$ & 0.95 & 0.90 & 0.85 & 0.8 \\
Proportion of Mild (M) that progress to Severe (H) & $\kappa_{i}$ & 0.03 & 0.06 & 0.09 & 0.12 \\
Proportion of Severe (H) that progress to Critical (C) & $\zeta_{i}$ & 0.1 & 0.13 & 0.16 & 0.19 \\
Proportion of Critical(C) that progress to Severe (H) & $\phi_{i}$ & 0.35 & 0.25 & 0.15 & 0.05 \\
Reciprocal of the average incubation period & $\omega$ & 0.2 & 0.2 & 0.2 & 0.2 \\
Recovery proportion of Asymptomatic (A) & $\gamma_{A, i}$ & 1 & 1 & 1 & 1 \\
Recovery proportion of Severe (H) & $\gamma_{H, i}$ & 0.9 & 0.87 & 0.84 & 0.81 \\
Recovery proportion of Mild (M) & $\gamma_{M, i}$ & 0.97 & 0.94 & 0.91 & 0.88 \\
Proportion of fatalities of Critical (C) & $\lambda_{i}$ & 0.65 & 0.75 & 0.85 & 0.95 \\
\hline
\end{tabular}



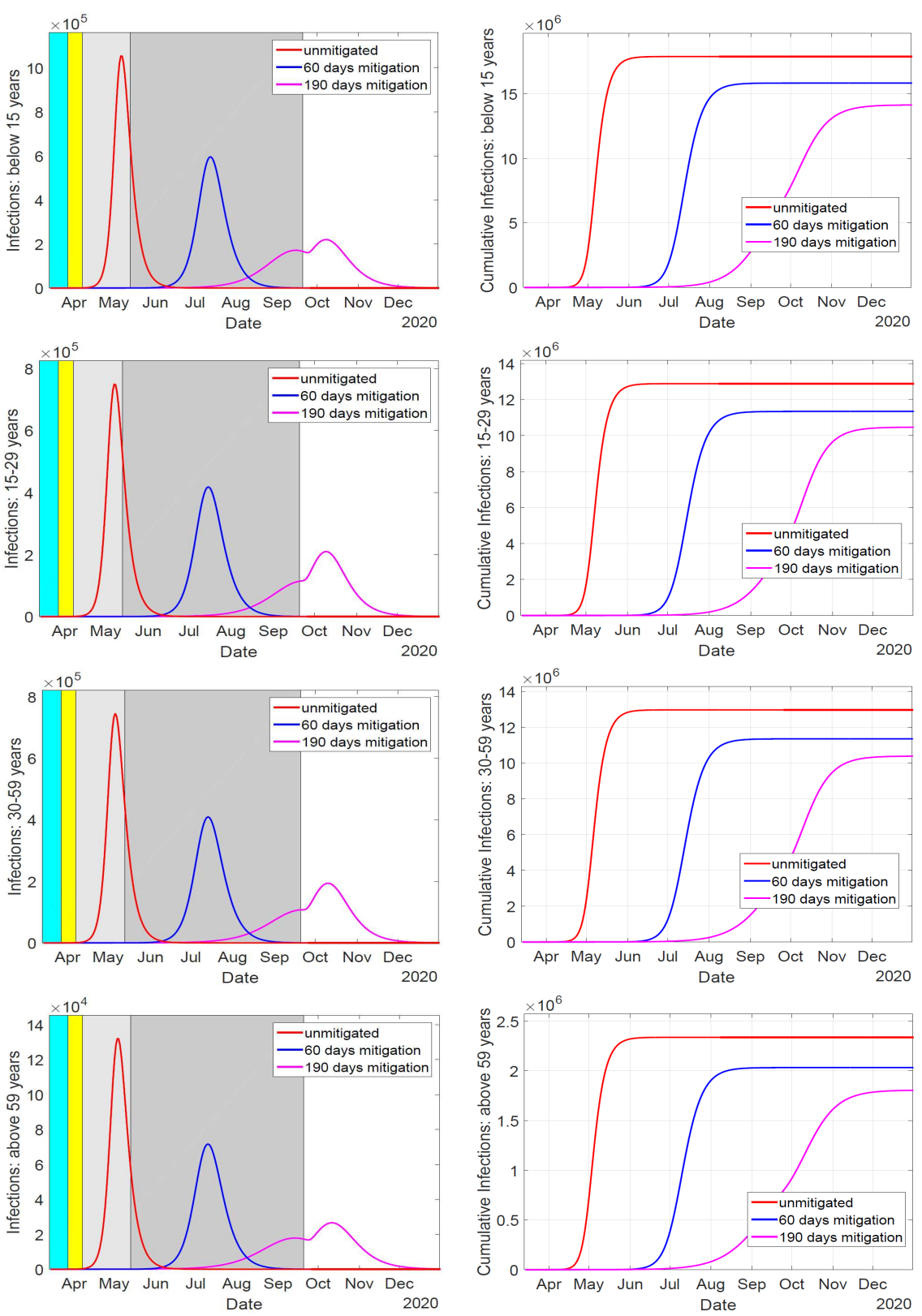

Figure 3 Effect of social distancing strategies on mitigating the spread of COVID-19 infections in Kenya. Applying the measures for up to $\mathbf{1 9 0}$ days, yielded a much significant reduction in epidemic as compared to the $\mathbf{6 0}$ days. The measures are quite effective in suppressing the disease transmission such that substantial levels of herd-immunity are not realized in the 60-days mitigation period, but in the 190-days mitigation period. This results to high number of infections in the 60-days mitigation once the measures are relaxed, while the herd-immunity realized in the 190-days period resolves the epidemic in the observed rise of infections after the measures are lifted. 
Table 2 Simulation outputs of the epidemic in Kenya in unmitigated and mitigated situations. Age-specific cumulative symptomatic, severe, critical and death cases are displayed. The peak of infections, in days, and peaks of demands for hospital and ICU beds, and deaths are also shown. The table also shows age-specific overall (symptomatic) attack rates, which are calculated as the number of infections (symptomatic cases) over the total population of that age band

\begin{tabular}{|c|c|c|c|c|}
\hline Age & Output & Unmitigated & 60-days mitigation & 190-days mitigation \\
\hline \multirow{10}{*}{ below 15 years } & Cumulative Symptomatic cases & 923,100 & 817,000 & 729,750 \\
\hline & Cumulative Severe cases & 27,749 & 24,559 & 21,937 \\
\hline & Cumulative Critical cases & 2,775 & 2,456 & 2,194 \\
\hline & Cumulative Deaths & 1,804 & 1,596 & 1,426 \\
\hline & Symptomatic Attack Rate & $4.97 \%$ & $4.40 \%$ & $3.93 \%$ \\
\hline & Overall Attack Rate & $96.33 \%$ & $85.26 \%$ & $76.15 \%$ \\
\hline & Infections Peak (days) & & 121 & 209 \\
\hline & Peak of Deaths & 103 & 59 & 22 \\
\hline & Peak of Hospital Beds demand & 13,360 & 12,044 & 14,452 \\
\hline & Peak of ICU Beds demand & 1,335 & 1,204 & 1,445 \\
\hline \multirow{10}{*}{$15-29$ years } & Cumulative Symptomatic cases & $1,368,300$ & $1,204,400$ & $1,111,300$ \\
\hline & Cumulative Severe cases & 79,296 & 69,798 & 64,406 \\
\hline & Cumulative Critical cases & 10,308 & 9,074 & 8,373 \\
\hline & Cumulative Deaths & 7,731 & 6,805 & 6,280 \\
\hline & Symptomatic Attack Rate & $10.27 \%$ & $9.04 \%$ & $8.34 \%$ \\
\hline & Overall Attack Rate & $96.61 \%$ & $85.04 \%$ & $78.47 \%$ \\
\hline & Infections Peak (days) & & 124 & 211 \\
\hline & Peak of Deaths & 436 & 248 & 125 \\
\hline & Peak of Hospital Beds demand & 33,903 & 32,700 & 39,623 \\
\hline & Peak of ICU Beds demand & 4,413 & 4,252 & 5,151 \\
\hline \multirow{10}{*}{$30-59$ years } & Cumulative Symptomatic cases & $2,117,800$ & $1,853,400$ & $1,696,100$ \\
\hline & Cumulative Severe cases & 176,420 & 154,390 & 141,290 \\
\hline & Cumulative Critical cases & 28,227 & 24,702 & 22,607 \\
\hline & Cumulative Deaths & 23,993 & 20,997 & 19,216 \\
\hline & Symptomatic Attack Rate & $15.89 \%$ & $13.91 \%$ & $12.73 \%$ \\
\hline & Overall Attack Rate & $97.23 \%$ & $85.09 \%$ & $77.87 \%$ \\
\hline & Infections Peak (days) & 54 & 123 & 211 \\
\hline & Peak of Deaths & 1341 & 749 & 356 \\
\hline & Peak of Hospital Beds demand & 79,473 & 75,008 & 86,043 \\
\hline & Peak of ICU Beds demand & 12,721 & 12,001 & 13,767 \\
\hline \multirow{10}{*}{ above 59 years } & Cumulative Symptomatic cases & 519,800 & 452,080 & 401,680 \\
\hline & Cumulative Severe cases & 55,039 & 47,869 & 42,531 \\
\hline & Cumulative Critical cases & 10,457 & 9,095 & 8,081 \\
\hline & Cumulative Deaths & 9,935 & 8,640 & 7,677 \\
\hline & Symptomatic Attack Rate & $21.84 \%$ & $18.99 \%$ & $16.88 \%$ \\
\hline & Overall Attack Rate & $98.19 \%$ & $85.40 \%$ & $75.88 \%$ \\
\hline & Infections Peak (days) & 52 & 119 & 212 \\
\hline & Peak of Deaths & 547 & 302 & 113 \\
\hline & Peak of Hospital Beds demand & 26,593 & 22,546 & 27,591 \\
\hline & Peak of ICU Beds demand & 4,475 & 4,284 & 5,242 \\
\hline
\end{tabular}


Figures

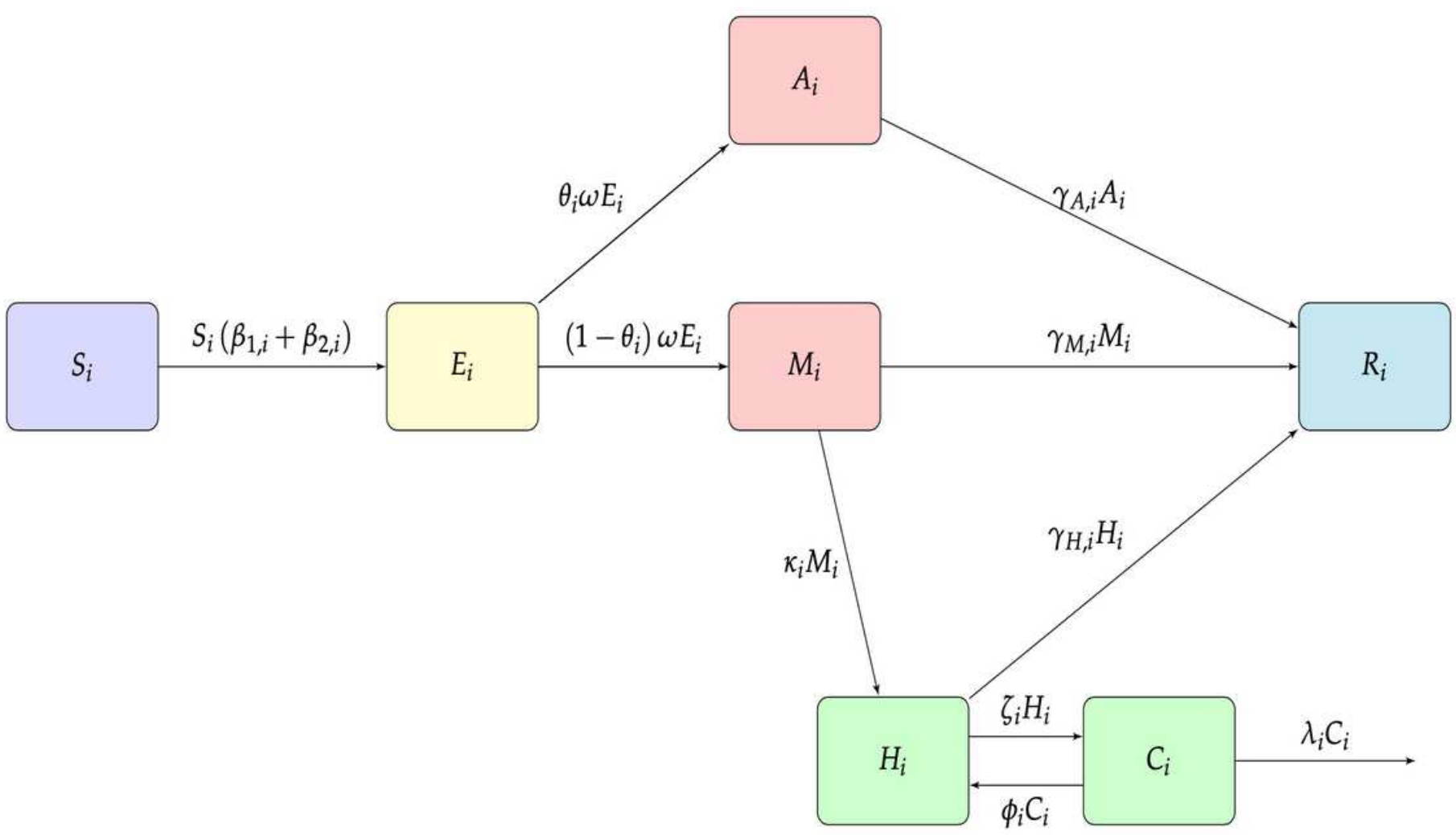

Figure 1

Flow diagram of the age-structured model for COVID-19 incorporating disease severity in the infected individuals 
Unmitigated

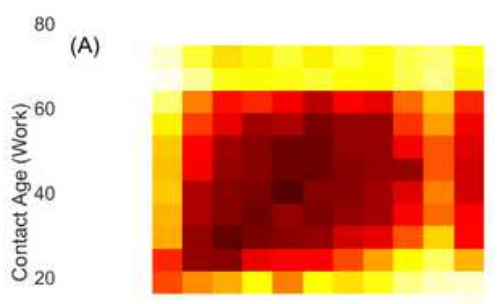

$\begin{array}{lllll}0 & 20 & 40 & 60 & 80\end{array}$

${ }^{80}$ (D)
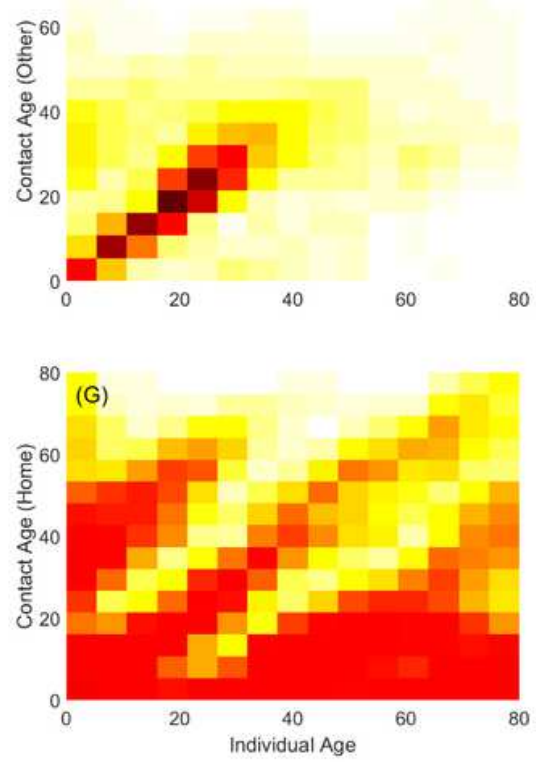

Curfew

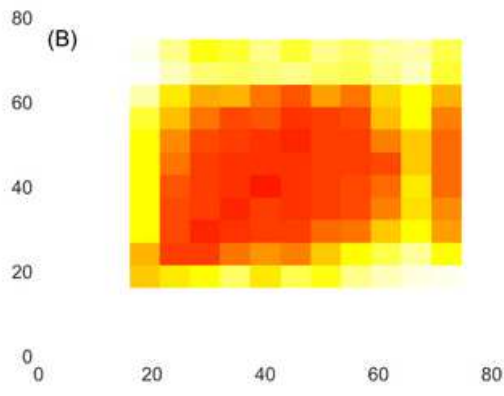

(E)
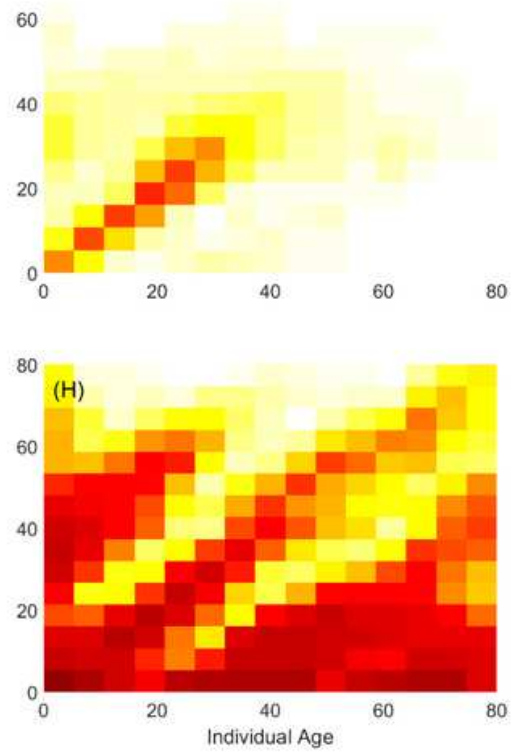

Restricted Movement

${ }^{80}$ (C)

60

20

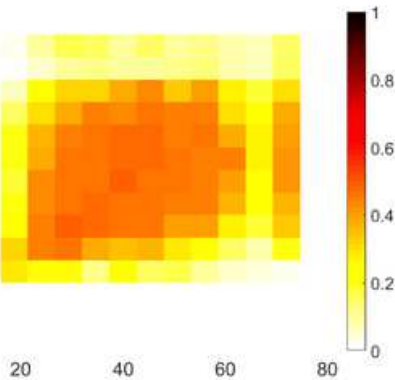

${ }^{80}(\mathrm{~F})$
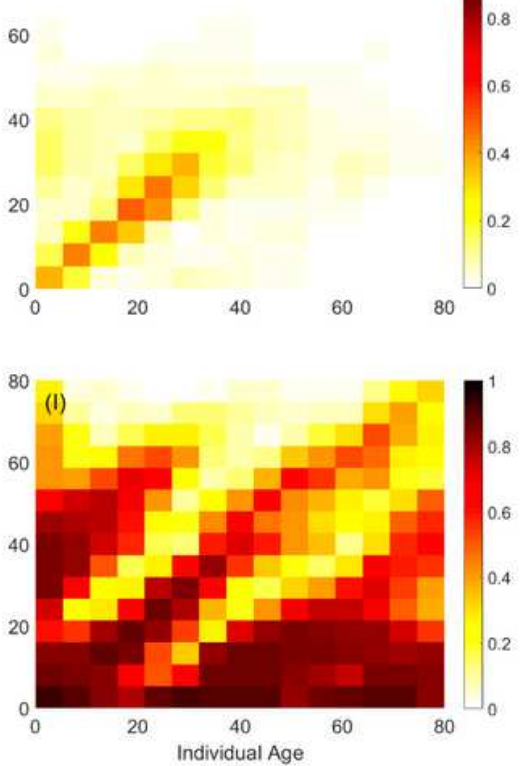

\section{Figure 2}

Effect of social distancing strategies on synthetic contact matrices for Kenya population (deduced from [?]). In the unmitigated scenario, there will be maximum contacts in workplaces and other locations (excluding home, schools and workplace). This results to less contacts at home. The dusk-to-dawn curfew results to a $35 \%$ reduction in contacts at workplaces and other locations, but assumed to increase the home contacts. The movement restriction yields a $45 \%$ reduction in contacts at workplaces and other locations, but presumed to increase the home contacts by $25 \%$. 

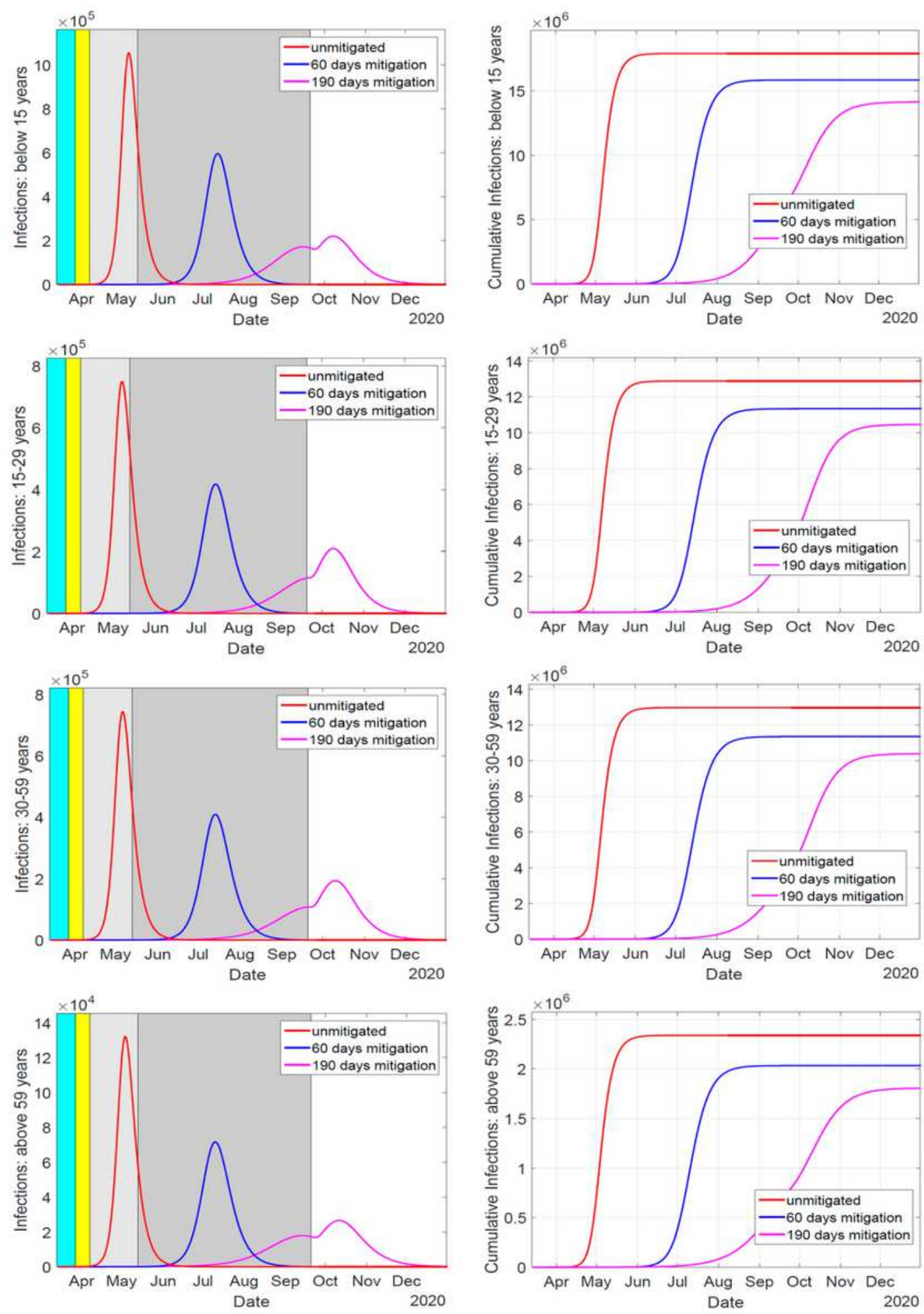

\section{Figure 3}

Effect of social distancing strategies on mitigating the spread of COVID-19 infections in Kenya. Applying the measures for up to 190 days, yielded a much significant reduction in epidemic as compared to the 60 days. The measures are quite effective in suppressing the disease transmission such that substantial levels of herd-immunity are not realized in the 60-days mitigation period, but in the 190-days mitigation period. This results to high number of infections in the 60-days mitigation once the measures are relaxed, 
while the herd-immunity realized in the 190-days period resolves the epidemic in the observed rise of infections after the measures are lifted. 